УДК 517.9

\title{
The Tensor Product and Quasiorder of an Algebra Related to Cohen-Macaulay Rings
}

\author{
Ali Molkhasi* \\ Department of Mathematics \\ University of Tabriz \\ Tabriz \\ Iran
}

Received 01.10.2014, received in revised form 10.11.2014, accepted 10.12.2014

This paper shows how the tensor products of the distributive lattices and the finite solvable groups can used to WB-height-unmixed of the method of Stanley and Reisner.

Keywords: quasiorder, polytopes, order complex, tensor product, distributive lattice.

\section{Introduction}

N. Funayama and T. Nakayama proves that congruence relations on an arbitrary lattice have an interesting connection with distributive lattices. For terminology and basic results of lattice theory and universal algebra see [3, 8], and [9]. By using distributive lattice and Stanley-Reisner theory, we formulate new characterizations of Cohen-Macaulay ring. Recall that the local ring $R$ is Cohen-Macaulay when so is $R$ as an $R$-module. A Noetherian ring (which may not be local) $R$ is said to be Cohen-Macaulay when its localization at any maximal ideal is Cohen-Macaulay local. In this paper relationships among quasiorder of an algebra, Cohen-Macaulay rings, the order complex of the lattice of all subgroups of a finite group and polytopes are considered. We denote the set of all quasiorders of an algebra $\mathbb{A}=(A, F)$, the set of all almost principal ideals of the lattice distributive $L$, the set of all almost principal filters of the lattice distributive $L$, and the lattice of all congruence relations of lattice $L$ by $\operatorname{Quord}(\mathbb{A}), \mathcal{I}(L), \digamma(L)$, and $\operatorname{Con}(L)$, respectively. Notice that $\mathbb{R}^{d}$ is the $d$-dimensional Euclidean space, $S \subseteq \mathbb{R}^{d}$ is a polytope and $K$ is a ring, and $E=K[S]$. In section 1 , it is proved that if $\mathbb{A}$ is an algebra with a majority term function, then rings $E[\operatorname{Quord}(\mathbb{A})]\left[X_{1}, X_{2}, \ldots\right]$ and $E[\digamma(\mathcal{I}(L))]\left[X_{1}, X_{2}, \ldots\right]$ are WB-height-unmixed. Also, if $\mathbb{A}$ is an algebra in any $n$-permutable variety, then $E[\operatorname{Con}(\mathbb{A})]\left[X_{1}, X_{2}, \ldots\right]$ is WB-height-unmixed. Finally in section 2 , it is shown that if $G$ is a finite solvable group and $\Re$ is the order complex of $L(G)$, the set of all subgroups of $G$, then $\Re\left[X_{1}, X_{2}, \ldots\right]$ is WB-height-unmixed. Finally, it is prove that if $C$ and $B$ are distributive lattices, then $E[C \otimes B]\left[X_{1}, X_{2}, \ldots\right]$ is WB-height-unmixed.

\section{Quasiorder of an algebra and the polytopes}

In this section relationship among quasiorder of an Algebra, the Cohen-Macaulay ring, and the polytopes are considered. Let we start the detailed investigation of the definition of a term function and quasiorder of an algebra. So, we recall some basic definitions.

*a-molkhasi@tabrizu.ac.ir

(c) Siberian Federal University. All rights reserved 
For an algebra $\mathbb{A}=(A, F)$, a term function $m\left(x_{1}, \ldots, x_{k}\right)$ is called a $k$-majority term (or sometimes, $k$-ary near unanimity term) if:

$$
m(y, x, x, \ldots, x)=m(x, y, x, \ldots, x)=\cdots=m(x, \ldots, x, y)=x
$$

holds for all $x, y \in A$. For instance, any lattice $(L, \wedge, \vee)$ admits a majority term. A quasiorder of an algebra $\mathbb{A}$ is a reflexive, transitive, binary relation which is compatible with the operations of $A$. Let $Q$ uord $(\mathbb{A})$ stand for the set of all quasiorders of $A$. It is easy to see that $($ Quord $(\mathbb{A}), \subseteq)$ is an algebraic lattice where the meet operation $\wedge$ is the set intersection $\cap$ of the binary relations. Let $L$ be an arbitrary lattice. Then $\operatorname{Con}(L)$, the lattice of all congruence relations of $L$, is distributive (Funayama and Nakayama) and $\operatorname{Con}(\mathbb{A})$ is an algebraic (Birkhoff and Frink 1948). Stanley-Reisner theory provides the central link between combinatorics and commutative algebra. In the following, we explain the basics of the Stanley-Reisner correspondence.

Definition 1.1. Let $R$ be a commutative ring, and $P$ be a finite poset(= partially ordered set), we say that $\mathfrak{A}$ is a ASL (algebra with straightening lows) on $P$ over $R$ if the followings hold:

ASL-0. An injective map $P \hookrightarrow \mathfrak{A}$ is given, $\mathfrak{A}$ is a graded $R$-algebra generated by $P$, and each element of $P$ is a homogeneous of positive degree. We call a product of elements of $P$ a monomial in $P$. In general, a monomial $M$ is a map $P \rightarrow \mathbb{N}_{0}$ and we denote $M=\prod_{x \in p} x^{M(x)}$ such that it also stands for an element of $\mathfrak{A}$. A monomial in $P$ of the form

$$
x_{i_{1}} \cdots x_{i_{l}}
$$

with $x_{i_{1}} \leqslant \cdots \leqslant x_{i_{l}}$ is called standard.

$A S L-1$. The set of standard monomials in $P$ is an $R$-free basis of $\mathfrak{A}$.

$A S L$-2. For $x, y \in P$ that $x \nless y$ and $y \nless x$, there is an expression of the form

$$
x y=\sum_{M} c_{M}^{x y} M\left(c_{M}^{x y} \in R\right)
$$

where the sum is taken over all standard monomials $M=x_{1} \cdots x_{r_{M}}\left(x_{1} \leqslant \cdots \leqslant x_{r_{M}}\right)$ with $x_{1}<x, y$ and $\operatorname{deg} M=\operatorname{deg}(x y)$.

The most simple example of an $A S L$ on $P$ over $\mathrm{R}$ is the Stanley-Reisner ring $\mathrm{R}[P]=\mathrm{R}[x \mid x \in$ $P] /(x y \mid x \nless y, y \nless x)$. The Stanley-Reisner rings play central role in theory of $A S L$.

We consider $\mathbb{R}^{d}$, the $d$-dimensional Euclidean space the points of which are $d$-tuples $x=$ $\left(\xi_{1}, \ldots, \xi_{d}\right)$ of real numbers, and the scalar product of which is given by

$$
\langle x, y\rangle=\sum_{i=1}^{d} \xi_{i} \eta_{i}, \quad x=\left(\xi_{1}, \ldots, \xi_{d}\right), \quad y=\left(\eta_{1}, \ldots, \eta_{d}\right) .
$$

A subset $K$ of $\mathbb{R}^{d}$ is convex, if for any two points $x_{0}, x_{1} \in K$ the line segment with end points $x_{0}$ and $x_{1}$, that is, the set of points $x=(1-\lambda) x_{0}+\lambda x_{1}, \lambda \in \mathbb{R}, 0 \leqslant \lambda \leqslant 1$, belongs to $K$. The intersection of any non-empty family of convex sets is again convex. This makes the definition of the convex hull, conv $X$, of a subset $X \subset \mathbb{R}^{d}$ to be the intersection of all convex sets $K \subset \mathbb{R}^{d}$ which contain $X$. The convex hull of $X$ can also be described as the set of all convex combinations of finite subsets of $X$, that is, as the set of linear combinations

$$
\lambda_{1} x_{1}+\ldots+\lambda_{r} x_{r}
$$

with

$$
x_{i} \in X, \quad \lambda_{i} \geqslant 0, \quad \sum_{i=1}^{r} \lambda_{i}=1
$$


Definition 1.2. A polytope is the convex hull of a finite set of points in $\mathbb{R}^{d}$.

Let $S \subseteq \mathbb{R}^{d}$ be a polytope such that its vertex set is $V=\left\{x_{1}, \ldots, x_{r}\right\} . \quad A$ subset $\left\{u_{1}, \ldots, u_{r}\right\} \subseteq V$ is called a face of $S$, if $S$ has a geometric face in which the vertices are $u_{1}, \ldots, u_{r}$. Let $K$ be a ring and define the ring

$$
K[S]=\frac{K\left[X_{1}, \ldots, X_{r}\right]}{I}
$$

where $K\left[X_{1}, \ldots, X_{r}\right]$ is the polynomial ring and $I$ is the ideal generated by the set of all monomials $X_{j_{1}}, \ldots, X_{j_{k}}$, in which the set $u_{j_{1}}, \ldots, u_{j_{k}}$ is not a face. The ring $K[S]$ is called the associated Stanley-Reisner ring of $S$ over $K$. It is proved in [2], that for any polytope $S$, this ring is Cohen-Macaulay, (see that reference for the basic definitions of Cohen-Macaulay rings).

In the following, we will investigate the relations among distributive lattice and WB-heightunmixed, and quasiorder of an algebra to establish the main theorem of this paper. Recall that the height of a prime ideal $P$ is the maximum length of the chains of prime ideals of the ring $\mathrm{R}$ of the following form,

$$
P_{1} \subset P_{2} \subset \cdots \subset P_{k}=P \text {. }
$$

We will denote the height of $P$ by $h t(P)$. An ideal $I$ of $\mathrm{R}$ is said to be height-unmixed, if all the associated primes of $I$ have equal height. That is $h t(P)=h t(Q)$, for all $P, Q \in A s s(I)$, where $A s s(I)$ denotes the set of associated primes of $I$. An ideal $I$ is said to be unmixed if there are no embedded primes among the associated primes of $I$. That is, $P \subseteq Q \Rightarrow P=Q$, for all $P, Q \in A s s(I)$. We will say that an ideal is WB-height-unmixed, if it is height-unmixed with respect to the set of weak Bourbaki associated primes and an ideal is WB-unmixed if it is unmixed with respect to the set of weak Bourbaki associated primes. A prime ideal $P$ is a weak Bourbaki associated prime of the ideal $I$ of the ring $\mathrm{R}$ if it is a minimal ideal of the form $I: a$, for some $a \in \mathrm{R}$. Let $\mathrm{R}$ be a Cohen-Macaulay ring. Then we know that $\mathrm{R}\left[X_{1}, X_{2}, \ldots\right]$ is Cohen-Macaulay ring. Now we are ready to present our main theorem.

Theorem 1.1. Let $\mathbb{A}=(A, F)$ be an algebra with a majority term function. Let $S \subseteq \mathbb{R}^{d}$ be a polytope, $K$ be a ring, and $E=K[S]$. Then the ring $E[Q u o r d(\mathbb{A})]\left[X_{1}, X_{2}, \ldots\right]$ is $W B$-heightunmixed.

Proof. Let $\alpha_{1}, \alpha_{2}, \alpha \in \operatorname{Quord}(\mathbb{A})$. It is known that the join $\alpha_{1} \vee \alpha_{2}$ in the lattice $\operatorname{Quord}(\mathbb{A})$ is the transitive closure of the union $\alpha_{1} \cup \alpha_{2} \subseteq A \times A$. Hence

$$
\alpha_{1} \vee \alpha_{2}=\bigcup\left\{\alpha_{i_{1}} o \ldots o \alpha_{i_{n}} \mid i_{1}, \ldots, i_{n} \in\{1,2\}, n \geqslant 1\right\} .
$$

Now, we prove that $\operatorname{Quord}(\mathbb{A})$ is distributive lattice.

$$
\begin{gathered}
\left(\alpha \wedge \alpha_{1}\right) \vee\left(\alpha \wedge \alpha_{2}\right)=\left(\alpha \cap \alpha_{1}\right) \vee\left(\alpha \cap \alpha_{2}\right)= \\
=\bigcup\left\{\left(\alpha \cap \alpha_{i_{1}}\right) o \ldots o\left(\alpha \cap \alpha_{i_{n}}\right) \mid i_{1}, \ldots, i_{n} \in\{1,2\}, n \geqslant 1\right\}= \\
=\alpha \cap\left(\bigcup\left\{\alpha_{i_{1}} o \ldots o \alpha_{i_{n}} \mid i_{1}, \ldots, i_{n} \in\{1,2\}, n \geqslant 1\right\}\right)=\alpha \wedge\left(\alpha_{1} \vee \alpha_{2}\right) .
\end{gathered}
$$

So, $(\operatorname{Quord}(\mathbb{A}), \wedge, \vee)$ is a distributive lattice. It is known that if the ring $\mathrm{R}$ is a Cohen-Macaulay ring and $P$ is a distributive lattice, then $\mathrm{R}[P]$ is a Cohen-Macaulay ring (see [14]). On the other hand, we have $S$ is a polytope. Consequently, $K[S]$ is a Cohen-Macaulay ring and Quord(A) is a distributive lattice (see [4]). Therefore, by applying Theorem 3.5 of [5], we can concluded that $E[\operatorname{Quord}(\mathbb{A})]$ is a Cohen-Macaulay ring. We also know if ring $\mathrm{R}$ is a Cohen-Macaulay ring, then $\mathrm{R}\left[X_{1}, X_{2}, \ldots\right]$ is WB-height-unmixed. Thus, $E[\operatorname{Quord}(\mathbb{A})]\left[X_{1}, X_{2}, \ldots\right]$ is WB-heightunmixed. 
Definition 1.3. A variety $V$ is said to have $n$-permutable congruences if every algebra from $V$ has $n$-permutable congruences.

Recall that Hagemann and Mitschke proved that a variety $V$ is $n$-permutable $(n \geqslant 2)$ if and only if there are ternary terms $m_{1}, \ldots, m_{n-1}$ such that $V$ satisfies the identities:

$$
\left\{\begin{array}{l}
x=m_{1}(x, z, z) \\
m_{i}(x, x, z)=m_{i+1}(x, z, z) \text { for all } i .
\end{array}\right.
$$

Theorem 1.2. Let $\mathbb{A}=(A, F)$ be an algebra in any $n$-permutable variety, and $S \subseteq \mathbb{R}^{d}$ be a polytope, $K$ be a ring, and $E=K[S]$. Then $E[C o n(\mathbb{A})]\left[X_{1}, X_{2}, \ldots\right]$ is WB-height-unmixed.

Proof. Well-known 2-permutability is just permutability and $n$-permutability implies $(n+1)$ permutability. Let $\mathbb{A}$ be an algebra in any $n$-permutable variety then every quasiorder of $\mathbb{A}$ is a congruence of $\mathbb{A}$, so $\operatorname{Quord}(\mathbb{A})=\operatorname{Con}(\mathbb{A})$ (see [16] and $[13]$ ), and $E[\operatorname{Con}(\mathbb{A})]\left[X_{1}, X_{2}, \ldots\right]$ is WB-height-unmixed.

\section{Tensor product of the distributive lattices and the finite solvable groups}

In this section we study property of a finite solvable group and tensor product of distributive lattices. For a finite group $G$, we will denoted by $L(G)$ the set of all subgroups of $G$ which it partially ordered by inclusion. Then $L(G)$ is a lattice, with respective meet and join operations $H \wedge K=H \cap K, \quad H \vee K=<H, K>$. The order complex $\Delta(\Pi)$ of a poset $\Pi$ is a set of chains of $\Pi$. Recall that a subset $C$ of $\Pi$ is a chain if any of elements of $C$ are comparable. Obviously, $\Delta(\Pi)$ is a simplicial complex.

Definition 2.1. A pure simplicial complex $\triangle$ is called shellable if the facets of $\triangle$ can be given a linear order $F_{1}, \ldots, F_{m}$ in such a way that $\left\langle F_{i}\right\rangle \cap\left\langle F_{1}, \ldots, F_{i-1}\right\rangle$ is generated by a non-empty set of maximal proper faces of $\left\langle F_{i}\right\rangle$ for all $i, 2 \leqslant i \leqslant m$ (see [10] and [2]).

It is clear that the following implications hold for simplicial complexes:

$$
\text { shellable } \Longrightarrow \text { Cohen-Macaulay. }
$$

We have $\mathrm{G}$ is solvable if and only if the order complex of $L(G)$ is (nonpure) shellable (see [1] and [19]). Now we have the following theorem:

Theorem 2.1. Let $G$ be a finite solvable group and $\Re$ be the order complex of $L(G)$. Then $\Re\left[X_{1}, X_{2}, \ldots\right]$ is WB-height-unmixed.

The concepts of almost principal ideals and filters will be important in our investigations. Let us recall that an ideal $\mathbf{I}$ of a lattice $L$ is said to be almost principal if its intersection with every principal ideal of $L$ is a principal ideal of $L$. If $L$ has a largest element, then every almost principal ideal is principal. In general, there are almost principal ideals which are not principal (see [12] or [15]). The notions of principal filter and almost principal filter are defined dually. The whole lattice $L$ is also regarded as an (almost principal) ideal and filter. The almost principal ideals have been first considered in the context of semilattices in [11]. Their relevance for affine completeness of distributive lattices and Stone algebras has been established in [15] and [10], respectively. Let $\mathcal{I}(L)$ and $\digamma(L)$ denote the sets of all almost principal ideals and almost principal filters of the lattice $L$, respectively. For every distributive lattice $L$, the set $\mathcal{I}(L)$ ordered by the set inclusion is again a distributive lattice. In fact, it is a sublattice of the lattice $I(L)$ of all ideals of $L$. Dually, we can consider $L$ as a sublattice of the distributive lattice $\digamma(L)$. Note that the natural ordering of $\digamma(L)$ is given by the inverse set inclusion: $F_{1} \leqslant F_{2}$ iff $F_{1} \supseteq F_{2}$. So we proved that the following theorem: 
Theorem 2.2. Let $S \subseteq \mathbb{R}^{d}$ be a polytope, $K$ be a ring, and $E=K[S]$. Then $E[\digamma(\mathcal{I}(L))]\left[X_{1}, X_{2}, \ldots\right]$ is WB-height-unmixed.

A principal element is an element that is both meet-principal and join-principal or $A \wedge E=$ $(A: E) E$ and $A E: E=A \vee(0: E)$, for all $A \in L$. Here, the residual quotient of two elements $A$ and $B$ is denoted by $A: B$, so $A: B=\vee\{X \in L \mid X B \leqslant A\}$. Also a lattice $(L, \wedge, \vee)$ is called principal lattice if each of its elements is principal and it called distributive lattice if $a \wedge(b \vee c)=(a \wedge b) \vee(a \wedge c)$ for all $a, b, c \in L$ (see [6]). Let $R$ be a commutative ring with identity. Then $L(R)$ is a principal lattice, if and only if, $R$ is a Noetherian multiplication ring and Cohen-Macaulay ring.

Corollary 2.1. Let $R$ be a commutative ring with identity, $\mathbb{A}=(A, F)$ be an algebra with a majority term function, and $L(R)$ be a principal lattice. Then $R[$ Quord $(\mathbb{A})]\left[X_{1}, X_{2}, \ldots\right]$ is WB-height-unmixed.

Corollary 2.2. Let $R$ be a commutative ring with identity, $\mathbb{A}=(A, F)$ be an algebra in any $n$-permutable variety, and $L(R)$ be a principal lattice. Then $R[C o n(\mathbb{A})]\left[X_{1}, X_{2}, \ldots\right]$ is $W B$ height-unmixed.

The uniqueness of a tensor product is clear from its definition as a solution of a universal problem. The tensor product of $C$ and $B$ is denoted by $C \otimes B$ and the image of $(c, b)$ under the canonical bihomomorphism $f: C \times B \longrightarrow C \otimes B$ is written as $c \otimes b$.

Theorem 2.3. Let $C$ and $B$ be distributive lattices. Also, let $S \subseteq \mathbb{R}^{d}$ be a polytope, $K$ be a ring, and $E=K[S]$. Then $E[C \otimes B]\left[X_{1}, X_{2}, \ldots\right]$ is WB-height-unmixed.

Proof. We know if the ring $\mathrm{R}$ is a Cohen-Macaulay ring and $P$ is a distributive lattice, then $\mathrm{R}[P]$ is a Cohen-Macaulay ring (see [14]). On the other hand, we have $S$ is a polytope. Consequently, $K[S]$ is a Cohen-Macaulay ring and $C \otimes B$ is a distributive lattice (Theorem 2.6 of [7]). Therefore, by applying Theorem 3.5 of [5], $E[C \otimes B]$ is a Cohen-Macaulay ring. Furthermore, we have if ring $\mathrm{R}$ is a Cohen-Macaulay ring, then $\mathrm{R}\left[X_{1}, X_{2}, \ldots\right]$ is WB-heightunmixed. Hence, $E[C \otimes B)]\left[X_{1}, X_{2}, \ldots\right]$ is WB-height-unmixed.

\section{References}

[1] M.Bruggesser, P.Mani, Shellable decompositions of cells and spheres, Math. Scand., 29(1971), 197-205.

[2] W.Bruns, J.Herzog, Cohen-Macaulay rings, Cambridge University Press, 1998.

[3] G.Birkhoff, Lattice theory, Amer. Math. Soc. Colloq. Publ., vol. 25, 1967.

[4] I.Chajda, S.Radeleczki, Congruence schemes and their applications, Comment. Math. Univ. Carolinae, 46(2005), no. 1, 1-14.

[5] C.De Concini, D.Eisenbud, C.Procesi, Hodge algebras, Asterisque, vol. 91, Societe Mathematique de France, Paris, 1982.

[6] R.P.Dilworth, Abstract commutative ideal theory, Pacific J. Math., 12(1962), 481-498.

[7] G.A.Fraser, The semilattice tensor product of distributive lattices, Tran. Amer. Math. Soc., 217(1976), 183-194.

[8] G.Gratzer, Universal algebra, D. Van Nostrand Company, Princeton, 1968. 
[9] G.Gratzer, Lattice theory. First concepts and distributive lattices, Freeman, San Francisco, Calif., 1971.

[10] M.Haviar, M.Ploscica, Affine complete Stone algebras, Algebra Universalis, 34(1995), $355-365$.

[11] K.Kaarli, L.Marki, E.T.Schmidt, Affine complete semilattices, Monatshefte Math., 99(1985), 297-309.

[12] K.Kaarli, A.F.Pixley, Polynomial Completeness in Algebraic Systems, Chapman and Hall/CRC, 2000.

[13] O.M.Mamedov, A.Molkhasi, On congruence shcemes and compatible relations of algebras, Transactions of National Academy of sciences of Azer., 4(2009), 101-106.

[14] A.Molkhasi, Polynomials, $\alpha$-ideals and the principal lattice, Journal of Siberian Federal University. Mathematics and Physics, 4(2011), no. 3, 292-297.

[15] M.Ploisicica, Affine complete distributive lattices, Order, 11(1994), 385-390.

[16] A.G.Pinus, I.Chajda, Quasiorders on universal algebras, Algebra Logika, 32(1993), no. 3, 308-325.

[17] M.Suzuki, On the lattice of subgroups of finite groups, Trans. Amer. Math. Soc., 70(1951), $345-371$.

[18] J.Usan, Congruences of n-group and of associated Hosszru-Gluskin algebras, Novi Sad. J. Math., 28(1998), 91-108.

[19] R.Woodroofe, An EL-labeling of the subgroup lattice, Proc. Amer. Math. Soc., 136(2008), $3795-3801$.

\section{Тензорное произведение алгебры отношений в кольцах Коэна-Маколея}

\section{Али Молкхаси}

Эта статья показывает, как тензорное произедение дистрибутивных решеток и конечных разрешимых групп можно использовать в WB несмешанной высоте метода Стенли и Райснера.

Ключевые слова: квазипорядок, политопы, порядковый комплекс, тензорное произведение, дистрибутивные решетки. 